\title{
INDEX TO SPECIFIC NAMES
}

abadima, 30, 192

abbotti, 235

abdera, 112

abrupta, 230

acara, 25, 84

acerata, 35, 235

acontias, 137

acrita, 29, 143

acritoides, 29, 140

acronycta, 190

actiaca, 128

acutipennis, 117

admatha, 23, 78

aequalis, 337

aethiops, 328

agema, 347

aglaonice, 20, 32, 186

alberta, 22, 307

albimaculata, 299

albomaculata, 190

alboradiata, 25, 97, 174

alciope, 37, 38, 322

alcippina, 38, 210

alicia, 33, 221, 323

althoffi, 33, 251

alticola, 350

ambigua, 29, 143

amicitiae, 20, 24, 40, 317

amphimalla, 161

amphiprotea, 331

anacreon, 21, 28, 198

anacreontica, 198

anaemia, 20, 30, 31, 177

andromache, 346

andromba, 68

anemosa, 25, 94

angolanus, 299

anomala, 350

ansorgei, 38,318

antinorii, 40, 316

apecida, 35,229

aquilia, 144 aquilina, 143

arabica, 24, 31, 73, 172

arcticincta, 94

arctifasciata, 331

areca, 28, 107

argentea, 36,281

artemisa, 182

asboloplintha, 20, 24, 196

asema, 26, 122

âstrigera, 26, 101

atergatis, 24, 30, 188

atolmis, 24, 27, 137

aubyni, 36, 304

aureola, 32, 142

aurivillii, 38, 323

axina, 31,180

balbina, 81

balina, 34, 217

banka, 33, 221

barberi, 25, 84

baumanni, 112

baxteri, 41, 42, 267

bella, 29, 144

bellona, 29, 144

bellua, 192

bendis, 182

beni, 29, 139

biraca, 230

bomba, 28, 198

bonasia, 33, 35, 220, 249

boseae, 312

braesia, 22, 24, 169

brahmsi, 35, 235

braunei, 210

brunnea, 26, 102

bukoba, 240, 336

burni, 24, 68

buruensis, 346, 348

buschbecki, 35, 291

butleri, 341

büttneri, 26, 118 
buxtoni, 81, 239

byatti, 213

cabira, 35, 229

cabiroides, 35, 221

caecilia, 30, 182, 211

caffra, 26, 84

caldarena, 32, 161

calida, 204

calyce, 72

camaena, 20, 23, 82

candida, 171

caoncius, 174

cappadox, 221

carmentis, 327

castanea, 328

catori, 48

cephaea, 192, 349

cepheus, 26, 111, 239, 348

cerasa, 21, 54, 302

cerita, 23,55

chaeribula, 29, 153

chaeribulula, 144

chambezi, 28, 132

chilo, 22, 23, 25, 89

cinerea, 20, 22, 23, 307

circeis, 41, 42, 292, 297

clarei, 193

confluens, 249

confusa, 340

conjuncta, 38, 319

connexa, 240

conradti, 36, 289

corona, 45

cretacea, 37,323

crystallina, 89

cuva, 22, 50

cydonia, 322

cynthia, 221, 230

cynthius, 220, 249

daira, 38, 210

damii, 22, 50

decora, 138

defasciata, 174

dejana, 292

depunctella, 292

derubescens, 37, 281

detecta, 32,164

dewitzi, 36, 286

diavina, 235

dice, 59

diogenes, 23, 156 dircea, 161

disjuncta, 38, 321

dissociata, 196

dohertyi, 346,348

dorotheae, 327

doubledayi, 20, 31, 171, 174, 177,

180

drucei, 252

dubiosa, 94

egina, 24, 26, 27, 28, 106, 192

eginopsis, 112

ehmckei, 61

ella, 31, 179

emini, 102

етрияа, 122

encedon, 38, 209

encedonia, 210

entebbia, 336

entoria, 347

epidica, 40,46

eponina, 220, 221, 239

equatorialis, 31,177

ertli, 332

esebria, 39, 331

eugenia, 22,53

exalbescens, 36,281

excelsior, 33, 215

fallax, 337

felina, 118

fenelos, 268

fenestrata, 186

flava, 327

flavescens, 340

flavomaculatus, 229

fornax, 33, 309

fulleborni, 267

fulva, 210

fulvescens, 340

fumida, 204, 323

fumigata, 347

gaekwari, 171

gea, 327

gelonica, 269

goetzi, 33, 213

gracilis, 122

grosvenori, 42,276

guillemei, 27, 117

halali, 27, 128 
harrisoni, 27, 107

helvimaculata, 41, 269

hoeneli, 89

horta, 23,76

hova, 22, 60

humilis, 21, 305

hypatia, 182, 192

hypoleuca, 28, 92

igati, 22, 49

igola, 36, 42, 302

inaureata, 328

indica, 347

induna, 28, 198

infuscata, 210

insignis, 23,81

insularis, 40, 345

interjecta, 327

intermedia, 27, 31, 159

intermediana, 34, 240

interrupta, 94, 319

issoria, 350

iturina, 21, 57, 305

jacksoni, 332

jalema, 25, 84

janisca, 239

jodutta, 39, 327

johnstoni, 39,339

kaffana, 262

kakana, 23, 57

karschi, 34, 230

katana, 35, 227

kenia, 337

khara, 107

kilimandjara, 337

kraka, 21, 52

lactea, 156

lactimaculata, 41, 269

lamborni, 37, 62

latifasciata, 323

leona, 41, 278

leonina, 278

leucographa, 23, 79

leucopyga, 27, 157

leucosoma, 169

lia, 23, 67

liacea, 174

liberia, 239

limonata, 41, 293

lindica, 144 liszti, 157

littoralis, 29, 144

lobemba, 25, 97

lofua , 26, 127

lualabae, 29, 155

lumiri, 33, 219

lutealba, 319

luxi, 137

lycia, 38,210

lусоа, $39,42,336$

lycoides, 292

lygus, 190

macarina, 38, 322

machequena, 22, 66

maculiventris, 302

madhela, 71

mahela, 22, 71

mairessei, 36, 286

makupa, 42

manandaza, 64

manca, 29, 144

mandane, 298

manjaca, 239

mansya, 27, 134

maransetra, 64

marginata, 140

marmorata, 26, 105

marnois, 30, 184

masamba, 41, 42, 312

masaris, 332

masonala, 50

matuapa, 72

medea, 27, 107

media, 336

medoa, 107

melaina, 29, 139

melanosticta, 286

melanoxantha, 36, 288

melas, 239

menippe, 25, 83

metaprotea, 332

meyeri, 346, 348

mhondana, 72,84

mima, 31, 32, 167

minima, 217

mirabilis, 20, 216

mirifica, 19, 208

modesta, 174

moluccana, 346, 347

mombasae, 21, 43

monteironis, 332

mosana, 94 
msamwiae, 143

murcia, 82

mutata, 319

mycenaea, 84

mystica, 171

nandensis, 321

natalensis, 230

natalica, 30, 190, 192

nataliensis, 120

nebulosa, 347

necoda, 210

neobule, 23, 72

neluska, 161

nero, 161

newtoni, 35, 285

nia, 257

nidama, 50

nigrescens, 112

nigroapicalis, 264

niobe, 20, 24, 344

nohara, 27, 128

ntebiae, 292

nubilata, 332

nyassicola, 143

obeira, 24, 68, 302

oberthuri, 34, 249

obscura, 174

ochrascens, 34, 240

ochreata, 252

octobalia, 340

oenone, 346,347

ombria, 161

omrora, 26, 124

oncaea, $30,174,184$

onerata, 27,135

opis, 298

oppidia, 40, 278

oreas, 39,298

orestia, 40,305

orestina, 305

oreta, 263

orientis, 292

orina, $39,40,263$

orinata, 264

orineta, 264

oscari, 25, 91

pallidepicta, 257

parce, 346,348

parrhasia, 41, 42, 277

parrhoppidia, 278

pasiphaë, 107 pauperata, 144

pelasgius, 40, 269

pella, 347

pelopeia, 42,274

peneleos, 36, 41, 42, 268, 277, 286

penella, 36, 281

penelope, 36,281

pentapolis, 40, 46

percussa, 50

perenna, 26, 37, 261

periphanes, 29, 139

perrupta, 239

persephone, 107

pervia, 301

petraea, 26, 114

petrina, 114

pharsaloides, 37, 256

pharsalus, 37, 256

pheusaca, 112

pica, 319

piva, 68

planesium, 221

pollonia, 347

polydectes, 261

pomponia, 281

praeponina, 221

protea, 331

proteina, 340

pudora, 30, 182

pudorella, 20, 32, 163

pudorina, 28, 144

punctellata, 27, 129

pseudatolmis, 27, 129

pseudegina, 30, 192

pseudepaea, 252

pseudolycia, 26, 101

pseudoprotea, 331

quirina, 22, 59

quirinalis, 40, 308

rabbaiae, 21, 43

radiata, 38, 204, 211

rahira, 24, 202

rakeli, 204

ranavalona, 22,64

rangatana, 34,240

recaldana, 161

regalis, 31,169

reversa, 293

rhodesiana, 31, 32, 166

rhodina, 257, 293

rogersi, 37,61 
rohlfsi, 24, 136

rosa, 59

rosina, 22,89

rougeti, 34, 239

rowena, 35,227

rubescens, 24, 196

rubra , 41, 293

rubrofasciata, 252

rudolphina, 107

rüppeli, 312

safie, 40, 315

salambo, 37, 61

saluspha, 257

sambavae, 41, 314

sanderi, 346, 347

saronis, 108

satis, 23, 44

schecana, 323

seis, 23,72

semialbescens, 340

semifulvescens, 340

semipunctella, 293

semivitrea, 39, 300

sepia, 41, 269

serena, 220, 239

servona, 39, 41, 42, 286, 292

sganzini, 210

siabona, 221

sidamona, 84

siginna, 81

silacea, 319

silia, 312

smithii, 309

sokotrana, 72

sotikensis, 35, 227

speciosa, 28, 198

stenobea, $30,32,190$

strattipocles, 41, 42, 311

subfulva, 327

subochreata, 293

subserena, 34, 239

subsquamia, 267

sucepha, 112

suffusa, 319

supponina, 35,227

sykesi, 31,171

taborana, 114

telekiana, 340

tella, 38, 323

telloides, 252 tenebrosa, 293

tenella, 34, 235

tenelloides, 35, 221

terpsichore, 34, 239, 350

tescea, 84

thelestis, 46

thesprio, 262

tirika, 336

toruna, 341

transienda, 293

transita, 306

translucida, 36, 281

trimeni, 84

turna, 21, 26, 105

ufipana, 94

umbrata, 26, 30, 124, 192

umbrina, 182

umida, 29, 140

unimaculata, 21, 56

unipunctella, 293

urungensis, 94

usagarae, 211

usaramensis, 144

utengulensis, 144

uvui, 33,217

ventura, 34,240

venturina, 240

vesperalis, 40, 48

vesta, 346, 349

vestalina, 350

vestita, 350

vestoides, 350

vinidia, 35,235

violae, 346,348

violarum, 26, 120

vitrea, 37,281

viviana, 34, 233

vuilloti, 37, 257

welwitschii, 25, 97

wigginsi, 28, 206

wissmanni, 89

zaire, 291

zambesina, 75

zetes, 24,83

zethea, 84

zethes, 84

zidora, 107

zitja, 24, 33, 204

zonata, 2], 42 


\section{$2 \mathrm{BHL}$ Biodiversity Heritage Library}

1912. "INDEX TO SPECIFIC NAMES." Transactions of the Entomological Society of London 60, 370-374. https://doi.org/10.1111/j.1365-2311.1912.tb03095.x.

View This Item Online: https://www.biodiversitylibrary.org/item/51237

DOI: https://doi.org/10.1111/j.1365-2311.1912.tb03095.x

Permalink: https://www.biodiversitylibrary.org/partpdf/14678

\section{Holding Institution}

Smithsonian Libraries

\section{Sponsored by}

Smithsonian

\section{Copyright \& Reuse}

Copyright Status: Public domain. The BHL considers that this work is no longer under copyright protection.

This document was created from content at the Biodiversity Heritage Library, the world's largest open access digital library for biodiversity literature and archives. Visit BHL at https://www.biodiversitylibrary.org. 